\title{
Sufentanil attenuates oxaliplatin cytotoxicity via inhibiting connexin 43-composed gap junction function
}

\author{
QI ZHANG and MIN SU
}

\author{
Department of Oncology, Hospital Affiliated to Hubei University of Arts and \\ Science/Xiangyang Central Hospital, Xiangyang, Hubei 441021, P.R. China
}

Received May 10, 2016; Accepted March 27, 2017

DOI: $10.3892 / \mathrm{mmr} .2017 .6669$

\begin{abstract}
Comprehensive strategies for the treatment of colorectal cancer (CRC) have become increasingly important. One of the most important factors is pain relief. Therefore, patients with CRC are concurrently treated with analgesics and chemotherapeutic agents; however, the effects of analgesics on the therapeutic activity of chemotherapeutic agents remain largely unknown. The present study investigated the effects of three widely used analgesics in clinics: Fentanyl, remifentanil and sufentanil, on the cytotoxicity of oxaliplatin, a commonly used chemotherapeutic agent for CRC. Furthermore, the underlying mechanisms of those effects in association with connexin 43 (Cx43)-composed gap junction (GJ) function were analyzed. The Lovo, Colo320, HCT116 and HT29 human CRC cell lines, with or without Cx43 expression, were used to examine the effects of the three analgesics on the cytotoxicity of oxaliplatin. The results demonstrated that in the cell lines expressing Cx43 (Lovo and Colo320), the cytotoxicity of oxaliplatin was attenuated and Cx43 GJ function was inhibited. Sufentanil, not fentanyl or remifentanil, inhibited Cx43 GJ function effectively, and reduced the cytotoxicity of oxaliplatin. In contrast, these effects were not observed in the other two colon cancer cell lines not expressing Cx43 (HCT116 and HT29). These results suggested that alternation of $\mathrm{Cx} 43 \mathrm{GJ}$ function may regulate the cytotoxicity of oxaliplatin in regard to CRC. Furthermore, sufentanil, not fentanyl or remifentanil, suppressed the cytotoxicity of oxaliplatin through inhibition of Cx43 GJ function. These results may be beneficial for the treatment of $\mathrm{CRC}$ and reduction of treatment resistance.
\end{abstract}

Correspondence to: Dr Min Su, Department of Oncology, Hospital Affiliated to Hubei University of Arts and Science/Xiangyang Central Hospital, 136 Jingzhou Street, Xiangyang, Hubei 441021, P.R. China

E-mail: hospitalren@163.com

Key words: colorectal cancer, connexin43, oxaliplatin, fentanyl, remifentanil, sufentanil

\section{Introduction}

Colorectal cancer (CRC) is a major public health problem accounting for $>1$ million cases of new cancer cases worldwide annually $(1,2)$. Although treatment strategies have improved in recent years, $\sim$ half a million patients still succumb to this disease every year (3). As a commonly used chemotherapeutic agent for CRC, oxaliplatin improves the response rate of patients and prolongs progression-free survival (4-6). However, oxaliplatin resistance is still a serious problem, the underlying mechanisms of which remain largely unknown.

Connexins (Cxs) are a large family of transmembrane proteins that exist in 21 isoforms expressed on all human organs and tissues. A total of six connexins compose a hemi-channel. Two hemi-channels dock together to form an integral gap junction (GJ) that regulates direct molecular transfer between the neighboring cells, including calcium, glutathione, cyclic adenosine monophosphate and cyclic guanosine monophosphate (molecules weighing $<1 \mathrm{kDa}$ ) (7). Molecules transferred via GJs are essential for many physiological and pathological events $(8,9)$. Cx43 is the most important of the Cx gene family, and has been reported to be associated with tumor progression and resistance to chemotherapeutic agents; for example, $\mathrm{Cx} 43$ GJ depression resulted in the resistance of temozolomide and cisplatin to glioblastoma and lung adenocarcinoma $(10,11)$. Therefore, the present study investigated the effects of $\mathrm{Cx} 43$ on the cytotoxicity of oxaliplatin in colon cancer cells, in order to confer a novel basis for therapies combating drug resistance.

Comprehensive strategies in the treatment of CRC have been developed for many years. One of the most important components is pain relief; cancer patients are often treated with analgesics and antineoplastic drugs concurrently to eliminate the pain resulted by cancers or the antineoplastic therapies $(12,13)$. However, the influence of analgesic agents on the antitumor activity of antineoplastic drugs has rarely been reported. The present study investigated the influence of commonly used analgesic agents, such as fentanyl, remifentanil and sufentanil, on the cytotoxicity of oxaliplatin, and the underlying mechanisms.

\section{Materials and methods}

Cell lines and cell culture. The Lovo, Colo320, HCT116 and HT29 human CRC cell lines (14) were obtained from the 
American Type Culture Collection (Manassas, VA, USA). Lovo was cultured in F-12K medium; Colo320 was cultured in RPMI-1640 medium; and HCT116 and HT29 were cultured in McCoy's 5a medium (Invitrogen; Thermo Fisher Scientific, Inc., Waltham, MA, USA). HeLa and HeLa-Cx43 cells (Sun Yat-sen University, Guangzhou, China) were cultured in Dulbecco's modified Eagle's medium (Invitrogen; Thermo Fisher Scientific, Inc.). All mediums were supplemented with $10 \%$ fetal bovine serum (FBS) and $100 \mathrm{U} / \mathrm{ml}$ penicillin-streptomycin (Invitrogen; Thermo Fisher Scientific, Inc.) in a $5 \% \mathrm{CO}_{2}$ incubator with $90 \%$ humidity at $37^{\circ} \mathrm{C}$ (Thermo Fisher Scientific, Inc.).

Colony-forming assay. A colony-forming assay was performed to determine cytotoxicity mediated by GJs. The cells were cultured at high- and low-density. At high density cell culture, cells were seeded at 100,000 cells $/ \mathrm{cm}^{2}$. When cells were exposed to drugs, the cultures were confluent $80-100 \%$ and GJs were formed. At low density cell culture, cells were seeded at 10,000 cells $/ \mathrm{cm}^{2}$. When cells were exposed to drugs, the cultures were not in contact with each other and GJs were not formed. Cells were treated with 0, 25, 50, 75, 100 or $125 \mu \mathrm{M}$ oxaliplatin (Sigma-Aldrich; Merck KGaA, Darmstadt, Germany) for $24 \mathrm{~h}$, and then washed with medium without FBS, harvested by trypsinization (Invitrogen; Thermo Fisher Scientific, Inc.), counted with a cell counting plate, diluted in medium containing FBS and penicillin-streptomycin, and seeded into 6-well plates at a density of 100 cells $/ \mathrm{cm}^{2}$. After 7 days, colony formation was assessed by staining with crystal violet (Sigma-Aldrich; Merck KGaA). Colonies containing more than 50 cells were scored $(6,15)$.

Chemical treatment. Cells were pretreated with a connexin mimetic peptide, gap26, for $1 \mathrm{~h}(300 \mu \mathrm{M}$; Sigma-Aldrich; Merck KGaA) prior to other assays to inhibit Cx43 GJ function. Dimethyl sulfoxide $(1 \mu \mathrm{l} / \mathrm{ml})$ served as the vehicle control (Sigma-Aldrich; Merck KGaA). All cell lines, including Lovo, Colo320, HCT116 and HT29, were treated for $24 \mathrm{~h}$ with $10 \mathrm{nM}$ fentanyl, $10 \mu \mathrm{M}$ remifentanil and $0.5 \mathrm{nM}$ sufentanil, which were all obtained from Yichang Humanwell Pharmaceutical Co., Ltd. (Yichang, China).

Parachute dye-coupling assay. Cx43 GJ function was detected by parachute dye-coupling assay in 24 -well plates. When cells grew into $80-100 \%$ confluent, cells were randomly selected in one well for use as donor cells. Donor cells were labeled with two different fluorescent dyes, CM-DiI ( $5 \mu \mathrm{M}$; Invitrogen; Thermo Fisher Scientific, Inc.) and calcein-acetoxymethyl ester (5 $\mu \mathrm{M}$, Invitrogen; Thermo Fisher Scientific, Inc.). CM-DiI did not spread to the neighboring cells, but calcein-acetoxymethyl ester stained coupled cells through Cx43 GJ. The cells were washed with medium without FBS and penicillin-streptomycin, harvested by trypsinization (Invitrogen; Thermo Fisher Scientific, Inc.), counted with cell counting plate, diluted with medium containing FBS and penicillin-streptomycin and seeded onto the receiver cells (80-100\% confluent, GJs formed) at a 1:150 donor/receiver ratio. A total of $4 \mathrm{~h}$ later, GJ function was examined under a fluorescence microscope (EclipseE800; Nikon Corporation, Tokyo, Japan). The mean number of receiver cells containing dye around the donor cell was counted and normalized to that of control cultures without any treatments (7).

Western blotting. Western blotting was performed as described previously $(16,17)$. Cells were washed three times with wash buffer [0.01 mol/1 PBS, $0.138 \mathrm{~mol} / 1 \mathrm{NaCl}, 0.02 \%$ $\mathrm{NaN} 3$ (pH 7.4)] and then lysed with $0.05 \mathrm{ml} / \mathrm{cm}^{2}$ lysis buffer for 2 h (Nanjing Keygen Biotech Co., Ltd., Nanjing, China) at $4^{\circ} \mathrm{C}$. Samples were centrifuged at $12,000 \mathrm{x} \mathrm{g}$ for $10 \mathrm{~min}$ at $4^{\circ} \mathrm{C}$. Protein concentrations were determined using the Bicinchoninic Acid method (Nanjing Keygen Biotech Co., Ltd.). Cell lysates $(25 \mu \mathrm{g})$ were separated by $10 \%$ SDS-PAGE (Invitrogen; Thermo Fisher Scientific, Inc.) and transferred onto a polyvinylidene difluoride membrane (Bio-Rad Laboratories, Inc., Hercules, CA, USA). Membranes were blocked with 5\% non-fat dry milk (Sigma-Aldrich; Merck $\mathrm{KGaA}$ ) at room temperature for $30 \mathrm{~min}$. Following this, the membranes were incubated with mouse monoclonal anti-human Cx43 (1:4,000; cat. no. C8093; Sigma-Aldrich; Merck KGaA) and anti- $\beta$-actin (1:10,000; cat. no. A1978; Sigma-Aldrich; Merck KGaA) antibodies overnight at $4^{\circ} \mathrm{C}$. After several washes with TBST (150 mM NaCL, $20 \mathrm{mM}$ Tris-HCL, $0.05 \%$ Tween-20), the membranes were incubated for $1 \mathrm{~h}$ at room temperature with a goat polyclonal anti-mouse IgG horseradish peroxidase (HRP)-conjugated secondary antibody (1:4,000; cat. no. M6898; Sigma-Aldrich; Merck KGaA). Protein bands were detected with an Enhanced Chemiluminescence system (KGP1125; Nanjing KeyGen Biotech. Co., Ltd.) and quantified using Alpha View software version 2.2.14407 (ProteinSimple; Bio-Techne, Minneapolis, MN, USA) $(16,17)$.

Statistical analysis. Statistical analysis was performed using SPSS 15.0 software (SPSS, Inc., Chicago, IL, USA). Multiple comparisons were analyzed using one-way analysis of variance, followed by Tukey's post hoc comparisons. $\mathrm{P}<0.05$ was considered to indicate a statistically significant difference. Data are presented as the mean \pm standard deviation.

\section{Results}

Oxaliplatin cytotoxicity varies in CRC cells with or without Cx43 channels. Lovo, Colo320, HCT116, HT29 CRC cells with or without $\mathrm{Cx} 43$ expression were used to investigate the effects of Cx43 channels on the cytotoxicity of oxaliplatin. The results of the present study were consistent with a previous study (14), in that Cx43 was expressed in the Lovo and Colo320 cell lines, but not in HCT116 or HT29 cells (Fig. 1A). A dye coupling assay demonstrated that Cx43 expressed on Lovo and Colo320 cells formed functional GJs (Fig. 1B). The four types of human CRC cell lines were cultured at low or high density and exposed to various concentrations of oxaliplatin. The cytotoxicity of oxaliplatin on Lovo (Fig. 1C) and Colo320 (Fig. 1D) cells at high density cell cultures (Cx43 expressed and GJs formed) was greater compared with low density cell cultures (Cx43 expressed, but no GJs formed). In contrast, this density-dependent cell cytotoxicity was not identified in HCT116 (Fig. 1E) or HT29 (Fig. 1F) cells (Cx43 not expressed and no GJs formed), which indicates that clonogenic survival had no difference at high or low density cell cultures. 

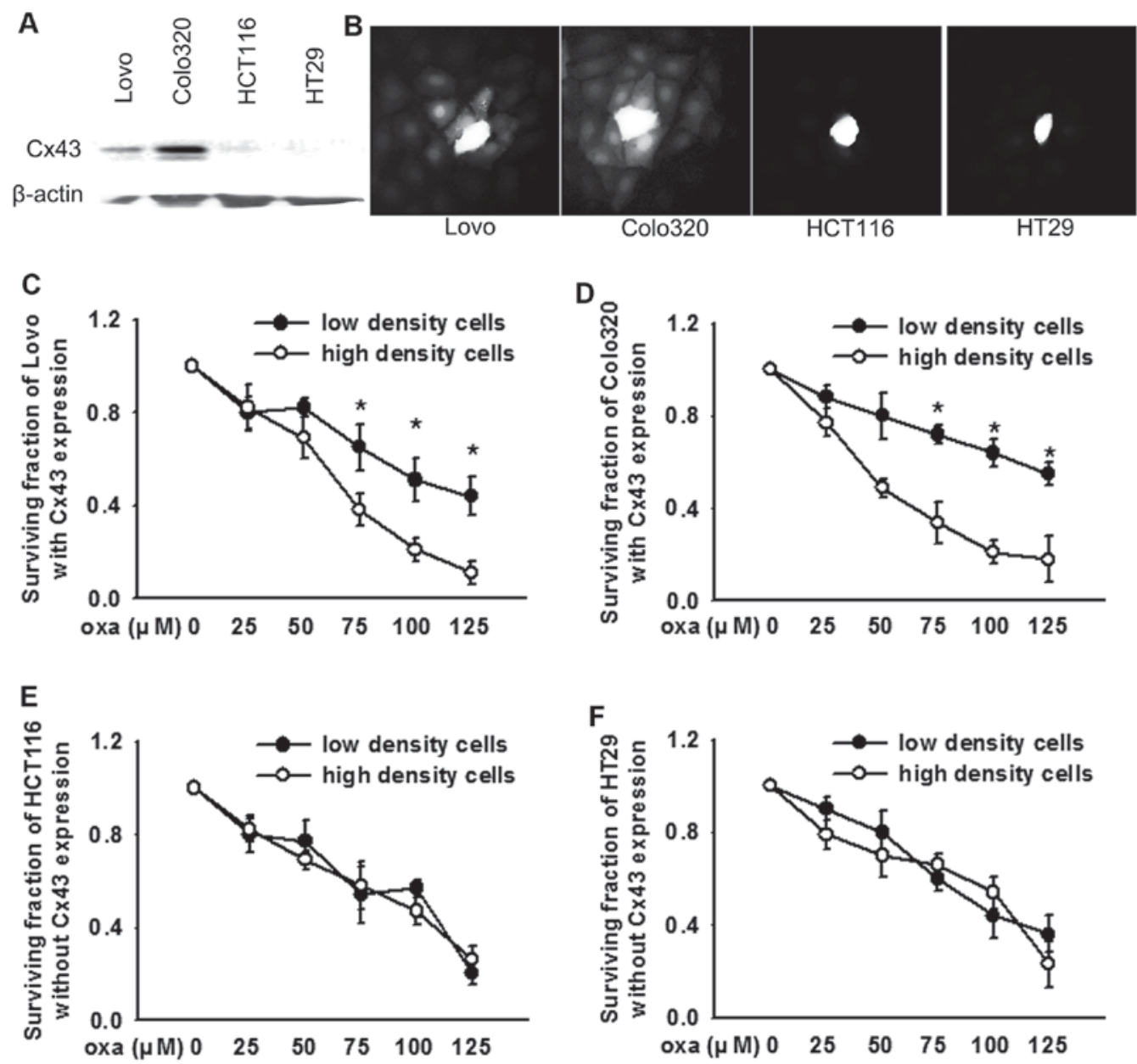

Figure 1. Oxaliplatin cytotoxicity varies in colon cancer cells with or without Cx43 channels. Cx43 (A) protein expression and (B) dye coupling in the Lovo, Colo320, HCT116 and HT29 cell lines. Survival of (C) Lovo, (D) Colo320, (E) HCT116 and (F) HT29 cells cultured at low or high density following exposure to oxaliplatin $(0-125 \mu \mathrm{M}, 24 \mathrm{~h}) . \mathrm{n}=5-7$; ${ }^{*} \mathrm{P}<0.05$ vs. high density cell culture group. Oxa, oxaliplatin; Cx43, connexin 43 . Data are presented as the mean \pm standard deviation.

Oxaliplatin cytotoxicity is mediated by Cx43 channels. Oxaliplatin cytotoxicity $(100 \mu \mathrm{M}, 24 \mathrm{~h})$ was attenuated in Lovo (Fig. 2A) and Colo320 (Fig. 2B) cells at high density cell culture ( $\mathrm{Cx} 43$ expressed and GJs formed), as $\mathrm{Cx} 43$ channel function was suppresed by gap26, a specific inhibitor of $\mathrm{Cx} 43$ channels. Although there was Cx43 expressed on Lovo and Colo320 cells, GJs did not form at low density cell cultures because cells made no contact with each other. Pre-treatment with gap26 did not alter the cytotoxicity of oxaliplatin. In HCT116 (Fig. 2C) and HT29 (Fig. 2D) cells, gap26 pre-treatment had no effects on the cytotoxicity of oxaliplatin in high or low density cell cultures, because there was no $\mathrm{Cx} 43$ expressed on the two cell lines. Therefore, Cx43 GJ function may contribute to the cytotoxicity of oxaliplatin.

Cx43 channel function may be attenuated by sufentanil, but not affected by fentanyl or remifentanil. HeLa cells, without any connexin protein expression, are frequently used to investigate connexin channel function by transfection with connexin proteins (6). The present study observed the effects of fentanyl, sufentanil and remifentanil on Cx43 GJ function in HeLa cells with or without Cx43 expression (Hela-Cx43, Cx43 expressed stably; Hela-WT, no Cx43 expressed; Fig. 3A). Dye transfer between Hela-Cx43 cells was clearer compared with
Hela-WT cells, which indicates that Hela-Cx43 cells formed functional GJs (Fig. 3B and C). The dye coupling assay also revealed that sufentanil suppressed $\mathrm{Cx} 43 \mathrm{GJ}$ function, and the inhibition rate was consistent with that of the $\mathrm{Cx} 43$ channel specific inhibitor, gap26. Fentanyl and remifentanil had no effects on dye transfer mediated by GJs composed of Cx43 (Fig. 3B and C).

Sufentanil attenuates oxaliplatin cytotoxicity via inhibiting Cx43 GJ function. As oxaliplatin cytotoxicity was demonstrated to be regulated by $\mathrm{Cx} 43$ channels in CRC cells (Fig. 2), and as sufentanil inhibited Cx43 GJ function (Fig. 3), it was hypothesized that sufentanil may affect oxaliplatin cytotoxicity in colon cancer cells via altering Cx43 GJ function. Therefore, the present study assessed oxaliplatin cytotoxicity in CRC cell lines with or without $\mathrm{Cx} 43$ expression when pretreated with fentanyl, sufentanil and remifentanil. After oxaliplatin exposure, cell growth of the four colon cancer cell lines Lovo, Colo320, HCT116 and HT29 were all reduced at both low density and high density cell cultures (Fig. 4A-D, respectively). However, the effects of fentanyl, sufentanil and remifentanil on oxaliplatin cytotoxicity in CRC cells with or without Cx43 expression were markedly different. Fig. 4A and B indicate that sufentanil pre-treatment 

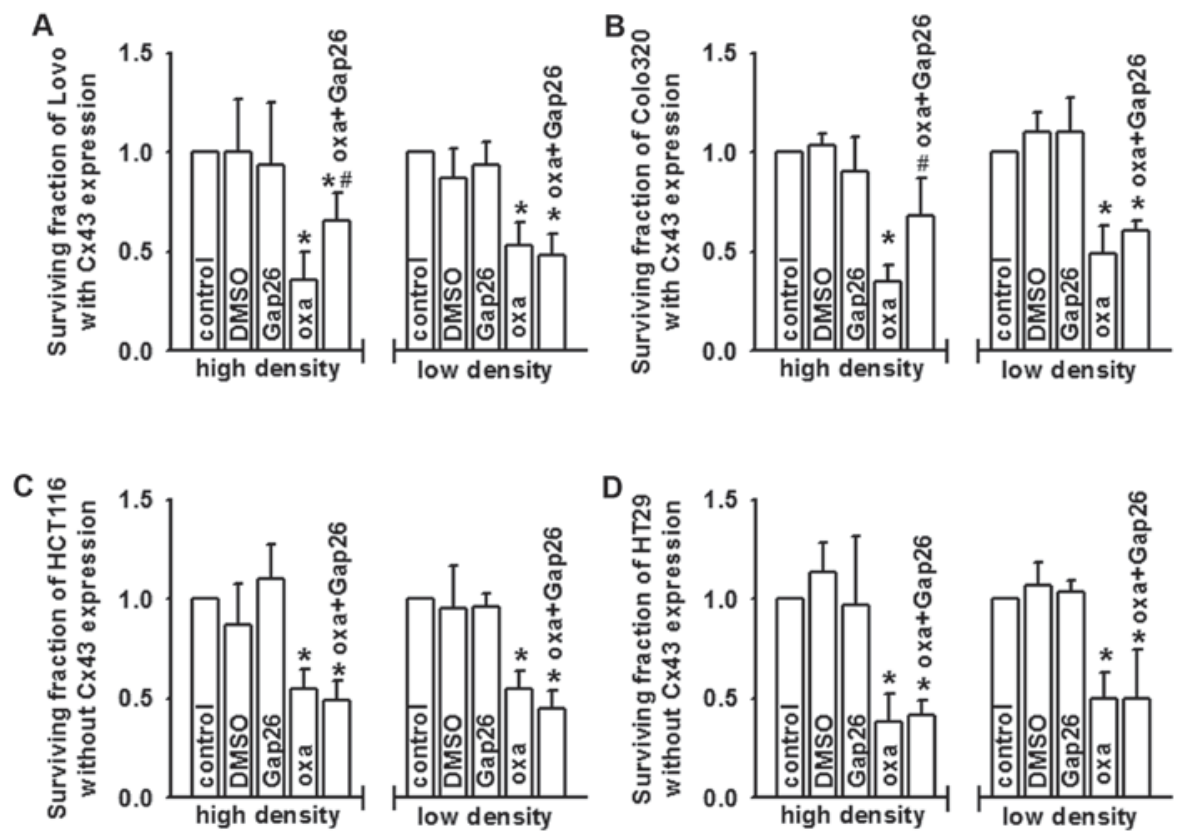

Figure 2. Effects of Gap26 on oxaliplatin cytotoxicity in colon cancer cells with or without Cx43 channels. Clonogenic survival of (A) Lovo, (B) Colo320, (C) HCT116 and (D) HT29 cells cultured at high or low density, exposed to oxaliplatin (100 $\mu \mathrm{M}, 24 \mathrm{~h}$ ) and co-incubated with or without gap26 (300 $\mu \mathrm{M}$, $1 \mathrm{~h})$. n=4-6; ${ }^{*} \mathrm{P}<0.05$ vs. control group; ${ }^{*} \mathrm{P}<0.05$ vs. oxa group. Oxa, oxaliplatin; DMSO, dimethyl sulfoxide; $\mathrm{Cx} 43$, connexin 43 . Data are presented as the mean \pm standard deviation.
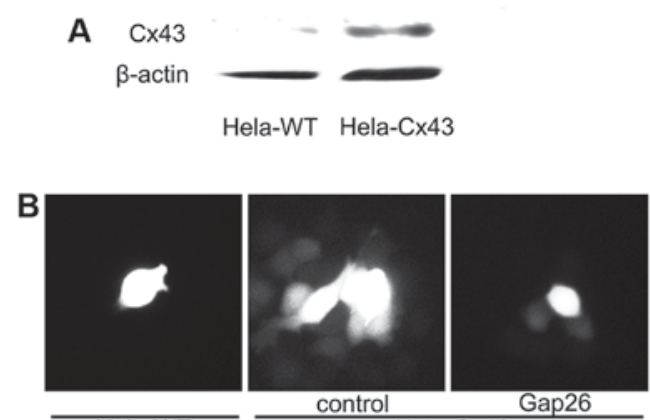

Hela-WT
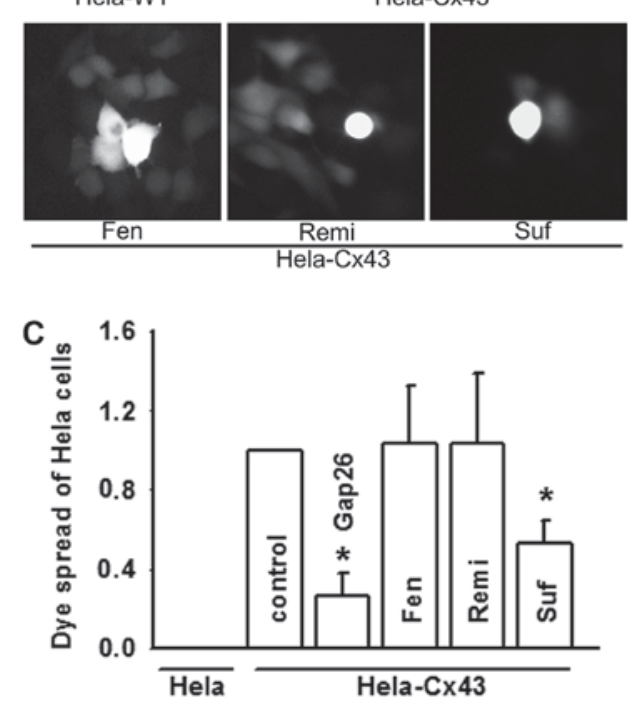

Figure 3. Sufentanil attenuates Cx43-composed gap junction function. (A) Cx43 protein expression in HeLa-WT and HeLa-Cx43 cells. (B) Fluorescence microscope images (magnification, x200) and (C) quantification of dye spread of HeLa cells following gap26, Fen, Remi and Suf treatment. $\mathrm{n}=5 ;{ }^{*} \mathrm{P}<0.05$ vs. control group of Hela-Cx43 cells. Suf, sufenatil; Fen, fentanyl; Remi, remifentanil; Cx43, connexin 43; Hela-Cx43, connexin 43 expressed stably; Hela-WT, no connexin 43 expressed. Data are presented as the mean \pm standard deviation. attenuated oxaliplatin cytotoxicity in Lovo and Colo320 cells at high density cell cultures (Cx43 expressed and GJs formed), as the survival fraction increase, but had no effects at low density cell cultures (Cx43 expressed, but functional GJs not formed). Fentanyl and remifentanil pre-treatment did not alter the cytotoxicity of oxaliplatin, at high density cell culture and low density cell cultures. However, cell density-dependent cytotoxicity was not observed in HCT116 (Fig. 4C) and HT29 (Fig. 4D) cells without Cx43 expression. No significant differences in oxaliplatin cytotoxicity were observed in high or low density cell cultures when pretreated with sufentanil (Fig. 4C and D).

\section{Discussion}

The present study investigated the influence of three analgesics, fentanyl, remifentanil and sufentanil, on oxaliplatin cytotoxicity in various $\mathrm{CRC}$ cell lines with or without $\mathrm{Cx} 43$ expression. The results demonstrated that in CRC cell lines with Cx43 expression (Lovo and Colo320), oxaliplatin exerted its effects in a cell density-dependent manner, as the survival fraction was much lower in high density cell cultures compared with in low density cell cultures. More importantly, sufentanil attenuated oxaliplatin cytotoxicity by inhibiting $\mathrm{Cx} 43$ channel function, but fentanyl and remifentanil had no effect. In contrast, in CRC cell lines without Cx43 expression (HCT116 and HT29), fentanyl, remifentanil and sufentanil had no any influence on oxaliplatin cytotoxicity in high and low density cell cultures. This investigation lead to the hypothesis that some analgesics commonly used concurrently with oxaliplatin or other antineoplastic agents in clinical settings, inhibited Cx43 GJ function and thereby attenuated the antineoplastic efficiency of oxaliplatin for tumors with $\mathrm{Cx} 43$ expression. Therefore, the choice of 

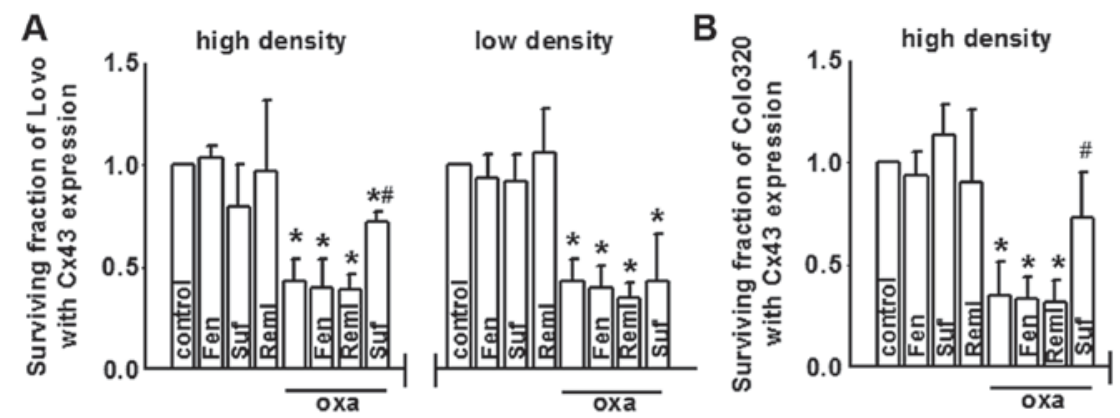

low density
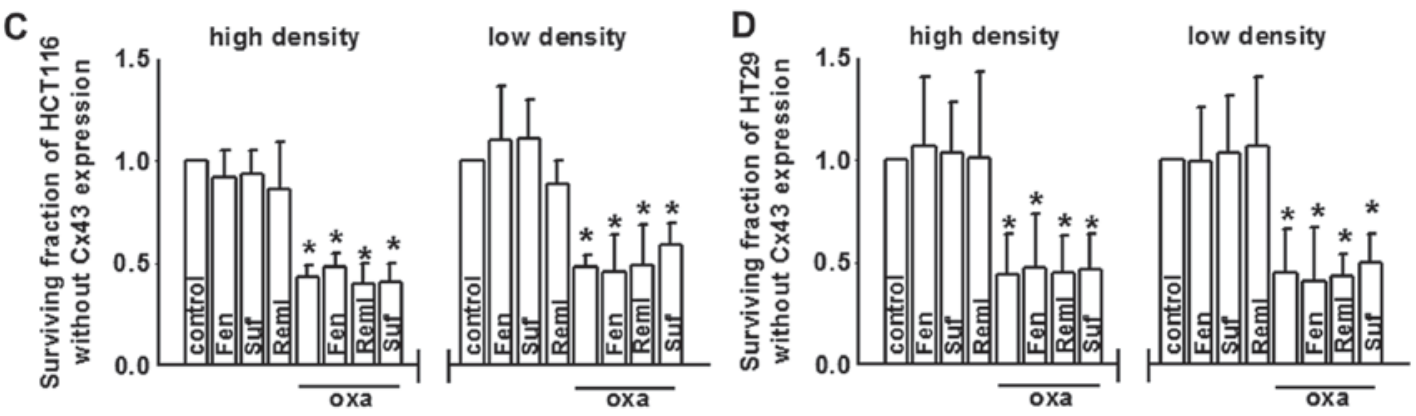

Figure 4. Effects of fentanyl, remifentanil and sufentanil on oxaliplatin cytotoxicity in colon cancer cells with or without Cx43 channels. Clonogenic survival of (A) Lovo, (B) Colo320, (C) HCT116 and (D) HT29 cells cultured at high or low density and exposed to oxaliplatin (100 $\mu$ M, 24 h) and co-incubated with Fen, Remi and Suf. $n=5-7 ;{ }^{*} \mathrm{P}<0.05$ vs. control group; ${ }^{\#} \mathrm{P}<0.05$ vs. oxa group. Suf, sufenatil; Fen, fentanyl; Remi, remifentanil; Cx43, connexin 43 . Data are presented as the mean \pm standard deviation.

analgesic for different cancer cells may impact the treatment effects of chemotherapeutic drugs, which should be considered by clinicians.

Until recently, CRC was one of the most important causes of cancer-associated mortality worldwide $(1,18)$. Although comprehensive strategies in CRC treatment have been improved for many years, the 5-year survival rate remains only $10 \%$ in patients with metastases $(19,20)$, the most important reason for which was the development of drug resistance during therapy $(21,22)$. However, the mechanisms of drug resistance remain largely unknown. Oxaliplatin, a third-generation platinum-based antineoplastic agent, is commonly used for CRC treatment. Its application improves the response rate and prolongs progression-free survival of patients with metastases. However, $\sim 40 \%$ patients develop resistance $(23,24)$. The present study identified a potential mechanism of oxaliplatin resistance, in that inhibition of $\mathrm{Cx} 43 \mathrm{GJ}$ function attenuated the cytotoxicity of oxaliplatin. Loss of $\mathrm{Cx} 43$ is common in the development of cancers; its deficiency contributes to the drug resistance $(25,26)$. It has previously been reported that $\mathrm{Cx} 43$ suppression results in temozolomide and cisplatin resistance in the treatment of glioblastoma or lung adenocarcinoma (10). The 'bystander effect' mediated by GJ is used to explain the mechanisms of drug resistance. Chemotherapy drugs attack cancer cells directly and lead to cell death. Notably, the attacked cells generate various toxic products, termed 'death signals', which are transferred between neighboring cells through GJs. 'Death signals' not only attack neighboring cells directly, but also activate different signal pathways, relative with cytotoxicity or apoptosis $(15,27)$. This effect amplifies the cytotoxicity of chemotherapy drugs. The results of the present study supported this conclusion that inhibition of Cx43 GJ function attenuates the cytotoxicity of oxaliplatin. Therefore, $\mathrm{Cx} 43$ expression recovery may represent an effective way to resolve drug resistance.

Notably, in the present study, a commonly used anesthetic in clinical anesthesia and intensive care unit sedation, sufentanil, inhibited GJ function and attenuated the cytotoxicity of oxaliplatin in CRC lines with Cx43 expression, but fentanyl and remifentanil had no effect. This issue should be considered by clinicians, because all of the three analgesics are currently extensively used for the management of pain; cancer patients are often treated concurrently with antineoplastic drugs and analgesics. Fentanyl, remifentanil and sufentanil interact with opioid receptors, and remifentanil and sufentanil selectively target the $\mu$ opioid receptor belonging to the $\mathrm{G}$ protein-coupled receptor family, which is considered to be one of the most significant protein families due to their importance as therapeutic targets (28). G protein-coupled receptors are involved in ligand recognition and subsequent activation or inactivation, because of their most essential characteristic, conformational flexibility $(28,29)$. Compared with fentanyl and remifentanil, sufentanil is highest affinity agonist targeting the $\mu$ opioid receptor (30). This suggests that sufentanil and $\mu$ opioid receptors may activate downstream signaling pathways of $\mathrm{G}$ proteins, resulting in $\mathrm{Cx} 43 \mathrm{GJ}$ function alternation. However, this hypothesis should be clarified in the future studies.

In conclusion, the present study demonstrated that in CRC cells, especially with Cx43 expression, such as Lovo and Colo320, sufentanil treatment decreased the cytotoxicity of oxaliplatin via inhibiting GJs composed of $\mathrm{Cx} 43$. These results may be beneficial for the treatment of CRC and reduction of treatment resistance. 


\section{References}

1. Lee W, Belkhiri A, Lockhart AC, Merchant N, Glaeser H, Harris EI, Washington MK, Brunt EM, Zaika A, Kim RB and El-Rifai W: Overexpression of OATP1B3 confers apoptotic resistance in colon cancer. Cancer Res 68: 10315-10323,2008.

2. Hirschi B, Gallmeier E, Ziesch A, Marschall M and Kolligs FT: Genetic targeting of B-RafV600E affects survival and proliferation and identifies selective agents against BRAF-mutant colorectal cancer cells. Mol Cancer 13: 122, 2014.

3. Tan S, Peng X, Peng W, Zhao Y and Wei Y: Enhancement of oxaliplatin-induced cell apoptosis and tumor suppression by 3-methyladenine in colon cancer. Oncol Lett 9: 2056-2062, 2015

4. Choi JH, Won YW, Kim HS, Oh YH, Lim S and Kim HJ: Oxaliplatin-induced sinusoidal obstruction syndrome mimicking metastatic colon cancer in the liver. Oncol Lett 11: 2861-2864, 2016.

5. Fan F, Gray MJ, Dallas NA, Yang AD, Van Buren G II, Camp ER and Ellis LM: Effect of chemotherapeutic stress on induction of vascular endothelial growth factor family members and receptors in human colorectal cancer cells. Mol Cancer Ther 7: 3064-3070, 2008.

6. Wang Q, You T, Yuan D, Han X, Hong X, He B, Wang L, Tong X, Tao L and Harris AL: Cisplatin and oxaliplatin inhibit gap junctional communication by direct action and by reduction of connexin expression, thereby counteracting cytotoxic efficacy. J Pharmacol Exp Ther 333: 903-911, 2010.

7. Luo C, Yuan D, Li X, Yao W, Luo G, Chi X, Li H, Irwin MG, $\mathrm{Xia} Z$ and Hei Z: Propofol attenuated acute kidney injury after orthotopic liver transplantation via inhibiting gap junction composed of connexin 32. Anesthesiology 122: 72-86, 2015.

8. Graziano AC, Parenti R, Avola R and Cardile V: Krabbe disease: Involvement of connexin43 in the apoptotic effects of sphingolipid psychosine on mouse oligodendrocyte precursors. Apoptosis 21: 25-35, 2016.

9. Spagnol G, Kieken F, Kopanic JL, Li H, Zach S, Stauch KL, Grosely R and Sorgen PL: Structural studies of the Nedd4 WW domains and their selectivity for the connexin43 (Cx43) carboxyl terminus. J Biol Chem 291: 7637-7650, 2016.

10. Gielen PR, Aftab Q, Ma N, Chen VC, Hong X, Lozinsky S, Naus CC and Sin WC: Connexin43 confers Temozolomide resistance in human glioma cells by modulating the mitochondrial apoptosis pathway. Neuropharmacology 75: 539-548, 2013.

11. Le HT, Sin WC, Lozinsky S, Bechberger J, Vega JL, Guo XQ, Sáez JC and Naus CC: Gap junction intercellular communication mediated by connexin43 in astrocytes is essential for their resistance to oxidative stress. J Biol Chem 289: 1345-1354, 2014.

12. He B, Tong X, Wang L, Wang Q, Ye H, Liu B, Hong X, Tao L and Harris AL: Tramadol and flurbiprofen depress the cytotoxicity of cisplatin via their effects on gap junctions. Clin Cancer Res 15: 5803-5810, 2009.

13. Myers J and Shetty N: Going beyond efficacy: Strategies for cancer pain management. Curr Oncol 15 (Suppl 1): S41-S49 2008.

14. Sirnes S, Bruun J, Kolberg M, Kjenseth A, Lind GE, Svindland A, Brech A, Nesbakken A, Lothe RA, Leithe E and Rivedal E: Connexin43 acts as a colorectal cancer tumor suppressor and predicts disease outcome. Int J Cancer 131: 570-581, 2012.
15. Zhao Y, Liu B, Wang Q, Yuan D, Yang Y, Hong X, Wang X and Tao L: Propofol depresses the cytotoxicity of X-ray irradiation through inhibition of gap junctions. Anesth Analg 112: 1088-1095, 2011

16. Yuan DD, Chi XJ, Jin Y, Li X, Ge M, Gao WL, Guan JQ, Zhang AL and Hei ZQ: Intestinal injury following liver transplantation was mediated by TLR4/NF- $\mathrm{B}$ activation-induced cell apoptosis. Mol Med Rep 13: 1525-1532, 2016.

17. Yuan D, Sun G, Zhang R, Luo C, Ge M, Luo G and Hei Z: Connexin 43 expressed in endothelial cells modulates monocyte-endothelial adhesion by regulating cell adhesion proteins. Mol Med Rep 12: 7146-7152, 2015.

18. Arnold M, Sierra MS, Laversanne M, Soerjomataram I, Jemal A and Bray F: Global patterns and trends in colorectal cancer incidence and mortality. Gut 66: 683-691, 2017.

19. Alcindor $\mathrm{T}$ and Beauger $\mathrm{N}$ : Oxaliplatin: A review in the era of molecularly targeted therapy. Curr Oncol 18: 18-25, 2011.

20. Howells LM, Sale S, Sriramareddy SN, Irving GR, Jones DJ, Ottley CJ, Pearson DG, Mann CD, Manson MM, Berry DP, et al: Curcumin ameliorates oxaliplatin-induced chemoresistance in HCT116 colorectal cancer cells in vitro and in vivo. Int J Cancer 129: 476-486, 2011.

21. Ekblad L, Kjellström J and Johnsson A: Reduced drug accumulation is more important in acquired resistance against oxaliplatin than against cisplatin in isogenic colon cancer cells. Anticancer Drugs 21: 523-531, 2010.

22. Chen J, Huang XF, Qiao L and Katsifis A: Insulin caused drug resistance to oxaliplatin in colon cancer cell line HT29. J Gastrointest Oncol 2: 27-33, 2011.

23. Peng L, Zhu H, Wang J, Sui H, Zhang H, Jin C, Li L, Xu T and Miao R: MiR-492 is functionally involved in Oxaliplatin resistance in colon cancer cells LS174T via its regulating the expression of CD147. Mol Cell Biochem 405: 73-79, 2015.

24. To KK, Poon DC, Wei Y, Wang F, Lin G and Fu LW: Data showing the circumvention of oxaliplatin resistance by vatalanib in colon cancer. Data Brief 7: 437-444, 2016.

25. Segretain D, Decrouy X, Dompierre J, Escalier D, Rahman N, Fiorini C, Mograbi B, Siffroi JP, Huhtaniemi I, Fenichel P and Pointis G: Sequestration of connexin43 in the early endosomes: An early event of Leydig cell tumor progression. Mol Carcinog 38: 179-187, 2003.

26. Leithe E, Sirnes S, Omori Y and Rivedal E: Downregulation of gap junctions in cancer cells. Crit Rev Oncog 12: 225-256, 2006.

27. Sanson M, Marcaud V, Robin E, Valery C, Sturtz F and Zalc B: Connexin 43-mediated bystander effect in two rat glioma cell models. Cancer Gene Ther 9: 149-155, 2002.

28. Fossepre M, Leherte L, Laaksonen A and Vercauteren DP: On the modularity of the intrinsic flexibility of the $\mu$ opioid receptor: A computational study. PloS One 9: e115856, 2014.

29. Katritch V, Cherezov V and Stevens RC: Structure-function of the $\mathrm{G}$ protein-coupled receptor superfamily. Annu Rev Pharmacol Toxicol 53: 531-556, 2013.

30. Wu W, Wei N, Jiang CN, Cui S and Yuan J: Effects of sufentanil on human gastric cancer cell line SGC-7901 in vitro. Cent Eur J Immunol 39: 299-305, 2014. 